\title{
Methods for Improving Screening for Vascular Cognitive Impairment Using the Montreal Cognitive Assessment
}

\author{
Khush-Bakht Zaidi (i), Jill B. Rich, Kelly M. Sunderland, Malcolm A. Binns, \\ Linda Truong, Paula M. McLaughlin, Bradley Pugh, Donna Kwan, Derek Beaton, \\ Brian Levine, Demetrios J. Sahlas, Dariush Dowlatshahi, Ayman Hassan, \\ Jennifer Mandzia, The ONDRI Investigators, Angela K. Troyer, Richard H. Swartz.
}

\begin{abstract}
Background: Vascular cognitive impairment (VCI) post-stroke is frequent but may go undetected, which highlights the need to better screen cognitive functioning following a stroke. Aim: We examined the clinical utility of the Montreal Cognitive Assessment (MoCA) in detecting cognitive impairment against a gold-standard neuropsychological battery. Methods: We assessed cognitive status with a comprehensive battery of neuropsychological tests in 161 individuals who were at least 3-months post-stroke. We used receiver operating characteristic (ROC) curves to identify two cut points for the MoCA to maximize sensitivity and specificity at a minimum 90\% threshold. We examined the utility of the Symbol Digit Modalities Test, a processing speed measure, to determine whether this additional metric would improve classification relative to the MoCA total score alone. Results: Using two cut points, $27 \%$ of participants scored $\leq 23$ and were classified as high probability of cognitive impairment (sensitivity $92 \%$ ), and $24 \%$ of participants scored $\geq 28$ and were classified as low probability of cognitive impairment (specificity $91 \%$ ). The remaining $48 \%$ of participants scored from 24 to 27 and were classified as indeterminate probability of cognitive impairment. The addition of a processing speed measure improved classification for the indeterminate group by correctly identifying $65 \%$ of these individuals, for an overall classification accuracy of $79 \%$. Conclusions: The utility of the MoCA in detecting cognitive impairment post-stroke is improved when using a three-category approach. The addition of a processing speed measure provides a practical and efficient method to increase confidence in the determined outcome while minimally extending the screening routine for VCI.
\end{abstract}

RÉSUMÉ : Des méthodes pour améliorer le dépistage des déficits cognitifs d'origine vasculaire au moyen de l'Évaluation cognitive de Montréal. Contexte : Bien que fréquents à la suite d'un AVC, les déficits cognitifs d'origine vasculaire (DCV) peuvent toutefois passer inaperçus, ce qui met en évidence la nécessité de mieux évaluer les fonctions cognitives des patients qui en sont victimes. Objectif : Nous nous sommes penchés sur l'utilité clinique de l'Évaluation cognitive de Montréal (MoCA) dans la détection des déficits cognitifs en comparaison avec une batterie de tests de référence. Méthodes : Nous avons évalué l'état cognitif de 161 patients au moyen d'une batterie exhaustive de tests neuropsychologiques. À noter qu'au moins 3 mois s'étaient écoulés depuis leur AVC. Nous avons utilisé des courbes caractéristiques de la performance d'un test (ROC curves) pour identifier deux seuils pathologiques (cut points) du MoCA, et ce, afin de maximiser sa sensibilité et sa spécificité à un seuil minimum de $90 \%$. Nous avons aussi examiné l'utilité du Symbol Digit Modalities Test, un test de vitesse cognitive de traitement, pour déterminer dans quelle mesure il nous permettrait d'améliorer la classification des patients par rapport au seul score total du MoCA. Résultats : En fonction de deux seuils pathologiques, $27 \%$ des participants ont obtenu $\leq 23$ et ont été considérés comme hautement susceptibles de souffrir de déficits cognitifs (sensibilité $92 \%$ ) alors que $24 \%$ des participants ont obtenu $\geq 28$ et ont été considérés comme étant très peu susceptibles de souffrir de ces mêmes déficits (spécificité $91 \%$ ). C'est donc dire que les autres participants (48\%) ont obtenu des scores de 24 à 27 . Il a été du coup impossible de déterminer la probabilité qu'ils soient atteints de déficits cognitifs. L'ajout du test de vitesse cognitive de traitement évoqué ci-dessus a permis d'améliorer la classification de ce groupe indéterminé en identifiant correctement $65 \%$ des individus, l'exactitude d'ensemble de la classification atteignant les $79 \%$. Conclusions : L'utilité du MoCA dans la détection de déficits cognitifs à la suite d'un AVC est bonifiée lorsqu' on utilise une approche dite des « trois catégories » (three-category approach). L'ajout du test Symbol Digit Modalities Test constitue en somme une méthode pratique et efficace pour augmenter la confiance dans un résultat déterminé tout en élargissant minimalement la routine de dépistage des DCV.

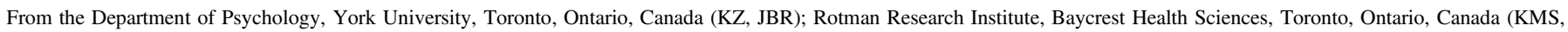

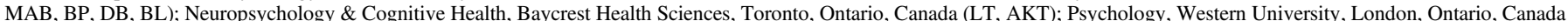

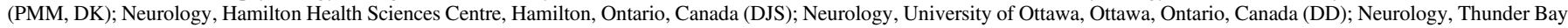

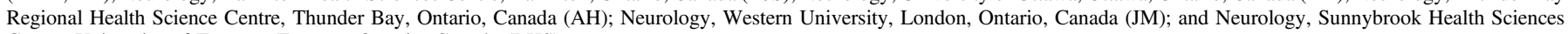
Centre, University of Toronto, Toronto, Ontario, Canada (RHS)

Received January 31, 2020. Final Revisions Submitted June 3, 2020. Date of Acceptance June 4, 2020.

Correspondence to: Khush-Bakht Zaidi, York University, 4700 Keele St, Toronto ON, M3J 1P3. Email: kzaidi@yorku.ca 
Cognitive impairment post-stroke can result in significant physical and psychological consequences for the individual. Stroke is one of the leading causes of adult disability, and in the past decade, hospitalization due to stroke-related cognitive impairment has increased by $35 \% .^{1}$ However, as many as $77 \%$ of individuals with stroke-related cognitive impairment may go undiagnosed by cognitive screening measures. ${ }^{2}$ The Montreal Cognitive Assessment (MoCA) ${ }^{3}$ is a widely used cognitive screening measure and has been recommended by the National Institute of Neurological Disorders and StrokeCanadian Stroke Network for use with stroke populations. ${ }^{4}$ Although cognitive impairment varies depending on stroke location and severity, several studies have found that individuals with cerebrovascular conditions are most likely to be impaired on MoCA items involving executive functions, fluency, recall, and attention..$^{5-7}$

Despite the MoCA's demonstrated use for cerebrovascular conditions, it lacks a processing speed metric, a function often affected in stroke populations. ${ }^{8,9}$ Speed of information processing can be easily assessed by tests that can be administered in under 2 minutes. The Symbol-Digit Modalities Test $\left(\right.$ SDMT) ${ }^{10}$ is a widely used test of processing speed that requires visual scanning, psychomotor speed, attention, and learning. Dong and colleagues ${ }^{11}$ demonstrated that when the MoCA was supplemented with the SDMT, it improved accuracy for vascular cognitive impairment (VCI) screening.

Traditionally, a single cut point of 26 is used on the MoCA to form dichotomous classifications of impaired or not impaired. ${ }^{3}$ An alternative method ${ }^{12-14}$ uses two cut points to classify individuals as low, indeterminate, or high probability of cognitive impairment. This allows for greater certainty of those classified as low or high probability of impairment, but leaves a subgroup whose classification is unknown and requires further assessment. Indeed, in the vascular sample studied by Swartz and colleagues ${ }^{14}$, a sizeable $62 \%$ scored in this indeterminate range.

\section{The Present Study}

In the present study, we sought to confirm and expand on previous findings of the clinical utility of the MoCA in detecting cognitive impairment using a diagnostic accuracy study design. First, we aimed to validate Swartz and colleagues, ${ }^{14}$ threecategory classification approach against a gold-standard neuropsychological assessment in a different sample of vascular pathology, post-acute ischemic stroke. A detailed comprehensive neuropsychological battery with standardized tests was used to assess and characterize cognitive impairment. ${ }^{3,4,17}$ Second, to improve diagnostic classification and reduce the number of people in the indeterminate category, we conducted two additional analyses. First, based on the literature showing specific deficits in executive function and processing speed in vascular pathology, ${ }^{5-7}$ we examined whether subdomain scores on the MoCA would provide additional predictive utility over and above the total score. Second, we included the SDMT with the MoCA to determine whether adding a simple, rapidly acquired measure of processing speed improved predictive utility. Screening conducted in the acute phase can result in reversible cognitive impairment, ${ }^{15}$ as such, we sought to examine cognition at a more stable time point post-stroke.

\section{METHOD \\ Participants}

This study was approved by the Research Ethics Boards at Baycrest Health Sciences and at York University. Participants were part of the Ontario Neurodegenerative Disease Research Initiative (ONDRI), a longitudinal, multidisciplinary research study investigating common profiles among five neurodegenerative conditions. ${ }^{16}$ The cerebrovascular disease (CVD) cohort was used in the present cross-sectional study. All participants completed various assessment platforms including genomics, neuroimaging, ocular function, and gait and balance, as well as neuropsychological testing. Demographic, clinical, and neuropsychological data obtained from the baseline assessment were used for this study. All participants were administered the MoCA as part of the screening procedure, and the neuropsychological battery (including the SDMT) was administered within 8 weeks of initial screening with the MoCA. If the participant had recently completed the MoCA for clinical purposes, efforts were made to administer alternate forms. Post-data collection, MoCA data underwent a rigorous cleaning and curation process that was overseen by a clinical neuropsychologist. Cleaning ensured that values were in range and that items were scored consistently. A working group of four individuals (including three neuropsychologists) highly familiar with the MoCA was established to reach a consensus and resolve any inconsistencies in scoring that required subjective judgment (i.e. Abstraction, Verbal Fluency). Neuropsychology data underwent rigorous monitoring and preprocessing. ${ }^{17}$ All ONDRI data underwent a data evaluation procedure using multivariate outlier detection to identify anomalous observations, providing guidance on additional areas of possible error. ${ }^{18}$

All participants provided informed consent and met extensive eligibility criteria for the larger ONDRI study. ${ }^{16}$ Participants in the CVD cohort were post-acute $\geq 3$ months and also met the following inclusion criteria: (a) proficient in speaking and understanding English, with self-ratings of 7 or more (corresponding to "good") for both speaking and understanding English on the Language Experience and Proficiency Questionnaire, ${ }^{19}$ (b) 8 or more years of formal education, (c) post-acute ischemic stroke or silent stroke that was documented on MRI or CT, (d) mildmoderate stroke severity defined by scores of $0-3$ on the modified Rankin Scale ${ }^{1}$ (MRS), ${ }^{20}$ and (e) a MoCA score of at least 18. Exclusion criteria included a history of dementia prior to the stroke, large cortical strokes, severe cognitive impairment, aphasia, inability to write, or severe functional disability limiting ability to perform the assessment. Data versions 2018SEP18 and 2018AUG17 were used for this study.

\section{Participant Characteristics}

Participant characteristics are provided in Table 1. There were 161 participants with $32 \%$ women and $68 \%$ men. Participants' age ranged from 54 to 85 years and were in their late $60 \mathrm{~s}$ on average and had some university education ( $M=14.6$ years). 


\section{Table 1. Participant Characteristics $(n=161)$}

\begin{tabular}{|c|c|c|c|c|}
\hline & $n$ & $M(S D)$ & Mdn & Range \\
\hline Age, years & & $69.2(7.4)$ & 68.9 & $54-85$ \\
\hline Education, years & & $14.6(2.9)$ & 14.0 & $8-20$ \\
\hline Sex ratio (male:female) & $110: 51$ & & & \\
\hline Individuals with subclinical stroke & $26(16 \%)$ & & & \\
\hline Time since stroke, months & & & 11 & $2-421$ \\
\hline Modified Rankin Score & & $1.0(0.8)$ & 1.0 & $0-4$ \\
\hline Total NIHSS & & $0.7(1.0)$ & 2.0 & $0-20$ \\
\hline MoCA score & & $25.2(3.0)$ & 26.0 & $18-30$ \\
\hline \multicolumn{5}{|l|}{ Symbol Digit Modalities Test } \\
\hline Coding & & $36.1(10.9)$ & 37.0 & $4-61$ \\
\hline Learning & & $6.5(4.5)$ & 6.0 & $0-15$ \\
\hline $\begin{array}{l}\text { Generalized Anxiety Disorder-7 } \\
\text { Scale }\end{array}$ & & $2.5(3.9)$ & 1.0 & $0-20$ \\
\hline $\begin{array}{l}\text { QIDS-SR - Depressed mood } \\
\text { domain }\end{array}$ & & $0.3(0.6)$ & 0.0 & $0-3$ \\
\hline
\end{tabular}

Note. MoCA = Montreal Cognitive Assessment; NIHSS = National Institutes of Health Stroke Scale; NP impairment = impaired status on neuropsychological battery; QIDS-SR = Quick Inventory of Depressive Symptoms - Self Report.

\section{Assessments}

\section{MoCA}

The MoCA is a screening tool that assesses multiple cognitive domains and is completed in under 10 minutes. $^{21}$ Individuals with $\leq 12$ years of education are given an additional point up to a maximum score of $30 .^{3}$

\section{Symbol-Digit Modalities Test}

The SDMT is a measure of processing speed and incidental learning. ${ }^{10}$ Participants are shown a key at the top of the page that pairs nine symbols with corresponding digits (1-9). They are then presented with rows of just symbols below for which they use the key to write the corresponding digit. The score is the total number of items filled in correctly in 90 seconds $(\max =110)$. Uchiyama and colleagues ${ }^{22}$ added an incidental learning component to the test featuring 15 symbols in which all 9 original symbols are included at least once. Participants are asked to fill in the number associated with each symbol from memory, without the assistance of the key, immediately following the coding trial. The score is the number of items recalled correctly $(\max =15)$. If a symbol is presented more than once and the participant responds correctly on one trial and incorrectly on another, they receive a point for the correct trial. Overall, two scores are obtained for the SDMT: coding and learning. For those with a physical impairment potentially impacting performance (e.g., tremor, paralysis, weakness, or injury), an oral version of the test is administered. Eight individuals (5\%) were administered the oral version of the SDMT, and the remaining $153(95 \%)$ completed the written version.

\section{Neuropsychological Assessment}

The neuropsychological assessment consisted of a standardized battery administered to all participants. For the purpose of
Table 2. Neuropsychological Battery

\begin{tabular}{l|l}
\hline Domain & Tests included \\
\hline Speed & $\begin{array}{l}\text { Symbol-Digit Modalities Test; Trail Making Test Parts A and B; } \\
\text { DKEFS Color-Word Interference Test Color Naming, Word } \\
\text { Reading, Inhibition, and Switching; DKEFS Verbal Fluency } \\
\text { Letters and Categories }\end{array}$ \\
\hline Memory & $\begin{array}{l}\text { Rey Auditory Verbal Learning Test Immediate Recall, Delayed } \\
\text { Recall, Recognition Hits; Brief Visuospatial Memory Test - } \\
\text { Revised Immediate Recall, Delayed Recall, Recognition } \\
\\
\text { Discrimination }\end{array}$ \\
\hline Visuospatial & $\begin{array}{l}\text { WASI-II Matrix Reasoning; Judgment of Line Orientation } \\
\text { (split-half); Visual Object and Space Perception Battery } \\
\text { Incomplete Letters }\end{array}$ \\
\hline Language & WASI-II Vocabulary; Boston Naming Test (15-item version); \\
& $\begin{array}{l}\text { Test of Adult Word Finding Verb Naming; Boston Diagnostic } \\
\text { Aphasia Examination-III Semantic Probe }\end{array}$ \\
\hline Attention & Wechsler Adult Intelligence Scale-III Digit-Span Forward, \\
& Backward, and Total
\end{tabular}

Note. DKEFS $=$ Delis-Kaplan Executive Functions System; WASI-II = Wechsler Abbreviated Scale of Intelligence - II.

characterizing areas of cognitive impairment in individual participants, the tests were categorized into five cognitive domains based on a principle component analysis as shown in Table 2. Test scores were normalized based on age and/or education using published norms and converted to standardized scores (z-scores, t-scores, scaled scores, and/or percentiles), in most cases. Participants were deemed cognitively impaired on the neuropsychological battery if they obtained a standardized score that was lower than 1.5 standard deviations (SDs) below the normative mean on at least two test scores within one or more domains. ${ }^{23}$ On two tests (SDMT incidental learning; Semantic Probe), normative data were 
not available, so we derived cutoff scores based on the available data while taking into consideration the test designs and estimated false-positive rates. To minimize fatigue, participants were offered breaks at scheduled intervals (approximately every 30-45 minutes of testing). Participants were permitted to take rest breaks as needed throughout the test session. In addition, participants were required to take a substantial break (i.e., at least 30 minutes) if testing continued for more than 3 hours. If any participant was unable to finish the testing in one session, additional sessions were scheduled on subsequent days. ${ }^{17}$

\section{Statistical Analyses}

Multiple linear regression analyses were performed to calculate the effects of age, education, sex, and depression on MoCA scores. To determine diagnostic accuracy, we identified individuals with a positive MoCA result (i.e., an impaired score, $<26$ ) versus a negative result (i.e., a normal score, $\geq 26$ ) and compared this to their performance on the neuropsychological tests. We then used receiver operating characteristic (ROC) curve analyses to maximize sensitivity and specificity to a) determine an optimal single cut point and b) to determine two cut points: the lowest value that achieved at least $90 \%$ sensitivity and the highest value that achieved at least $90 \%$ specificity. Participants scoring below the high sensitivity cut point were classified as high probability for cognitive impairment, and participants scoring above the high specificity cut point were classified as low probability. Participants scoring between the two cut points were classified as indeterminate or unknown probability of cognitive impairment. Positive and negative likelihood ratios, predictive values, and overall classification accuracies were calculated for all cut point analyses.

To improve classification for the indeterminate group, a second analysis was conducted using discriminant function analysis. ${ }^{24,25}$ A predictive model was built to correctly classify participants as impaired or not impaired as determined by their performance on the neuropsychological battery. We examined two sets of variables to create two separate discriminant functions. First, we examined the predictive value of distinct MoCA subdomain items, rather than the total MoCA score, to determine if the distributed weighting of subdomains would better predict group membership relative to the coarser single score. Second, we examined the utility of the SDMT, paired with the total MoCA score, to determine whether the additional metrics of processing speed and incidental learning would improve diagnostic accuracy relative to the MoCA total score alone. Participants from the high and low probability of cognitive impairment groups were used first as the learning data to develop the discriminant functions, and then the models were tested on the indeterminate group. The cross-validated classification method was used to indicate the final number of cases correctly identified by each function. This method employs the leave-one-out technique in which one case is systematically held out and the discriminant analysis is performed on the remaining sample. Then, the excluded case is classified into one of the groups based on the discriminant function and the procedure is repeated on each case of the sample until all cases are classified. This results in a more conservative estimate of the number of cases correctly identified by the function. ${ }^{25}$ All statistical analyses were conducted in SPSS Version 22.0.
Table 3. Number of Participants Impaired vs. Not Impaired on the Neuropsychology Battery as a Function of MoCA Score

\begin{tabular}{l|c|c}
\hline MoCA Score & Impaired $(\boldsymbol{n})$ & Not impaired $(\boldsymbol{n})$ \\
\hline 18 & 3 & 0 \\
\hline 19 & 7 & 0 \\
\hline 20 & 5 & 1 \\
\hline 21 & 4 & 0 \\
\hline 22 & 9 & 4 \\
\hline 23 & 11 & 4 \\
\hline 24 & 12 & 5 \\
\hline 25 & 10 & 8 \\
\hline 26 & 14 & 13 \\
\hline 27 & 12 & 13 \\
\hline 28 & 5 & 10 \\
\hline 29 & 3 & 8 \\
\hline 30 & 0 & 66 \\
\hline Total & 95 & 0 \\
\hline
\end{tabular}

Note. MoCA $=$ Montreal Cognitive Assessment.

\section{ReSUlts}

\section{Neuropsychological Data}

Of the 161 participants, $95(59 \%)$ met the criteria for cognitive impairment $(\geq 1.5$ SD below the normative mean on $\geq$ two test scores in at least one domain) on the neuropsychological battery. Forty-six participants (29\%) were impaired in two or more cognitive domains, and 6 (4\%) were impaired in three or more domains.

\section{MoCA Performance}

The mean MoCA score was 25.2 (3.0), with 75 (47\%) participants scoring below the traditional cut point of 26 and $86(53 \%)$ participants scoring at or above 26 . In a multiple regression model $\left(R^{2}=.069, p<.001\right)$ with age, sex, years of education, and depression as main effects, only age was a significant predictor of MoCA scores, where older participants $(\beta=-0.106, p<.001)$ had lower MoCA scores. Sex $(\beta=.068$, $p=.891)$, years of education $(\beta=.093, p=.243)$, and depression scores $(\beta=-.212, p=.596)$ did not significantly predict MoCA scores. Table 3 presents a count of the number of participants who were cognitively impaired or intact based on the neuropsychological battery as a function of MoCA score.

Next, we examined neuropsychological performance of the MoCA-negative $(\geq 26)$ and MoCA-positive $(\leq 25)$ groups. Of the 86 individuals in the MoCA-negative group, 34 (40\%) were impaired in at least one neuropsychological domain. Regarding the areas of impairment, $26(30 \%)$ participants demonstrated impaired speed, 19 (22\%) had impaired memory, 2 (2\%) had impaired visual spatial processing, 1 (1\%) had impaired language, and $1(1 \%)$ had impaired attention. Of these 34 who were impaired on at least one domain, 12 (35\%) were impaired in two or more domains, and $2(17 \%)$ of those 12 participants were impaired in three or more domains on the neuropsychological battery. 
Table 4. Diagnostic Accuracy Analysis and Optimal Cut Points

\begin{tabular}{l|c|c|c}
\hline Diagnostic Characteristics & Traditional Cut Point $\geq \mathbf{2 6}$ & Single Optimal Cut Point $\geq \mathbf{2 7}$ & $\begin{array}{c}\text { Two Optimal Cut Points } \\
\text { Sensitivity: } \leq \mathbf{2 7} \\
\text { Specificity: } \mathbf{2 4}\end{array}$ \\
\hline Sensitivity & $64 \%$ & $79 \%$ & $92 \%$ \\
\hline Specificity & $79 \%$ & $67 \%$ & $91 \%$ \\
\hline PPV & $81 \%$ & $77 \%$ & $89 \%$ \\
\hline NPV & $60 \%$ & $69 \%$ & $79 \%$ \\
\hline +LR & 3.02 & 2.37 & 5.42 \\
\hline -LR & 0.45 & 0.32 & 0.18 \\
\hline Individuals correctly identified & $113(70 \%)$ & $119(74 \%)$ & $76 / 83(92 \%)$ \\
\hline
\end{tabular}

Note. $+\mathrm{LR}=$ positive likelihood ratio; $-\mathrm{LR}=$ negative likelihood ratio; NPV = negative predictive value; PPV = positive predictive value.

Of the 75 individuals in the MoCA-positive $(\leq 25)$ group, $61(81 \%)$ were impaired in at least one of the neuropsychological domains. Thirty-nine (52\%) were impaired in speed, $49(65 \%)$ were impaired in memory, $10(13 \%)$ were impaired in language, $1(1 \%)$ was impaired in visual spatial, and no individual was impaired in the attention domain. Of these 75 individuals, $34(45 \%)$ were impaired in two or more domains, and $4(12 \%)$ of those 34 were impaired in three or more domains.

\section{Diagnostic Accuracy Analysis}

Using the traditional cut point of 26, the MoCA's sensitivity was $64 \%$ and specificity was $79 \%$ in the present sample. Our ROC analysis indicated that the optimal single cut point, which maximized sensitivity and specificity, was 27 . The optimal two cut points were 27 (to maximize sensitivity at 92\%) and 24 (to maximize specificity at $91 \%$ ). All results involving cut points are summarized in Table 4 . Using two cut points, $27 \%$ of participants scored $\leq 23$ and were classified as high probability for cognitive impairment, and $24 \%$ of participants scored $\geq 28$ and were classified as low probability for cognitive impairment. The remaining $48 \%$ of participants scored in the intermediate range of 24 to 27 and were classified as indeterminate probability for cognitive impairment. Figure 1 displays the proportion of individuals in each category and their cognitive status based on the neuropsychological assessment.

\section{Discriminant Function Analysis}

\section{MoCA subdomain scores}

Eight MoCA variables were created by grouping scorable items into their respective cognitive domains, including attention (10 points), immediate recall (10 points), delayed recall (15 points), visual construction (5 points), abstraction ( 2 points), fluency (25 points), object naming (3 points), and orientation (6 points). With participants from the high and low probability of cognitive impairment groups, the discriminant function using MoCA variables was significant, $\Lambda=.57, \chi^{2}(8)=43.67$, $p<.001$, accounting for $43 \%$ of between-group variability. The structure matrix correlation coefficients reveal the strength of each variable in the model with the function. Items of attention $(r=.61)$, immediate recall $(r=.34)$, delayed recall $(r=.55)$, language fluency $(r=.53)$, visual construction $(r=.48)$, abstraction $(r=.45)$, and orientation $(r=.30)$ were significant group predictors, whereas object naming was not, $r<.30 .^{25}$ The cross-validated classification showed that overall, 65 of $83(78 \%)$ cases in the high and low groups were correctly identified based on the predictors in the model. Next, in order to test the predictive utility of the model, we conducted a discriminant function analysis on the indeterminate MoCA group alone. The discriminant function for the indeterminate group was not significant, $\Lambda=.87, \chi^{2}(8)=10.15, p=.254$, and accounted for $13 \%$ of between-group variability.

\section{Symbol-Digit Modalities Test}

The SDMT was removed from the neuropsychological battery before conducting these analyses to avoid issues of circularity. There were $33(21 \%)$ individuals who were impaired on the SDMT in our full sample, and two participants no longer met criteria for cognitive impairment on the neuropsychological battery when the SDMT was removed.

We entered two subscores from the SDMT in the discriminant function analysis, a coding subscore (normative-based z-score) and a learning subscore (raw total score). Using the high and low probability of cognitive impairment groups, the discriminant function was significant, $\Lambda=.44, \chi^{2}(3)=65.01, p<.001$, accounting for $56 \%$ of between-group variability. Analysis of the structure matrix scores revealed that the MoCA total score $(r=.88)$, SDMT coding score $(r=.62)$, and SDMT learning score $(r=.52)$ were all significant group predictors. The crossvalidated classification showed that overall, 69 of 83 (83\%) cases were correctly identified. Next, we tested this model for the indeterminate group alone. This discriminant function analysis was also significant, $\Lambda=.88, \chi^{2}(3)=9.62, p<.022$, accounting for $12 \%$ of between-group variability. Analysis of the structure matrix revealed that the SDMT coding score, SDMT learning score, and the MoCA total score were all significant predictors of group membership for the indeterminate group, $r s=.89, .62$, and .51 , respectively. The cross-validated classification showed that overall, 51 of $78(65 \%)$ cases were correctly identified for this group. Thus, across the entire sample, 127/161 (79\%) cases were correctly identified as determined by performance on the larger neuropsychological battery. 


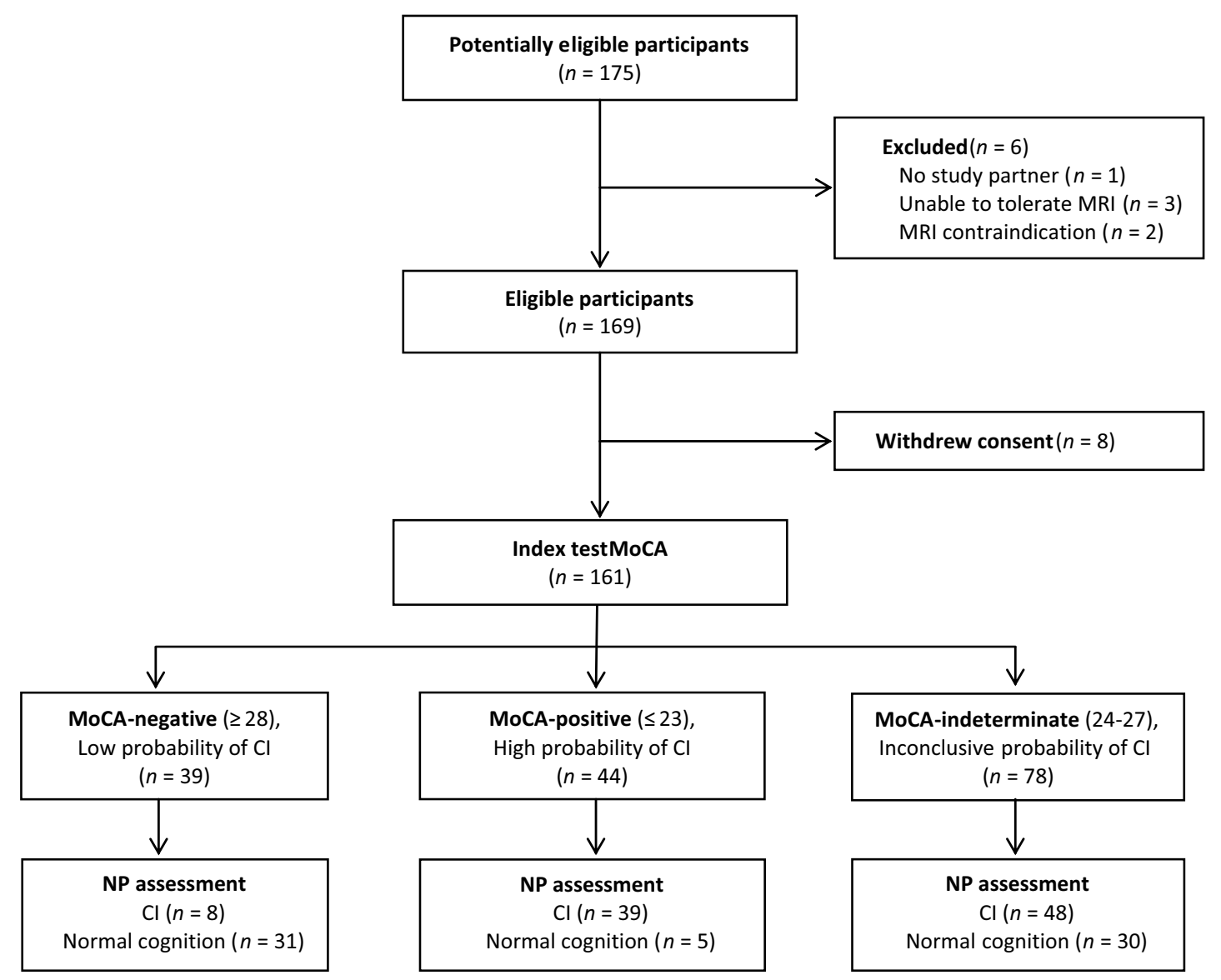

Figure 1: Flow Diagram of Participant Classification by the MoCA (Index) and Neuropsychological Assessment (Reference).

Abbreviations: CI, cognitive impairment; MoCA, Montreal Cognitive Assessment; NP, neuropsychological assessment.

\section{Discussion}

The present study evaluated the clinical utility of the MoCA by using a three-category approach to maximize classification for cognitive impairment and combining it with the SDMT. First, we tested two cut points that maximize the MoCA's sensitivity and specificity to higher than $90 \%$ for post-acute stroke individuals. This means that the cognitive status determined by performance on the neuropsychological battery was correctly identified by using the MoCA total score for more than $90 \%$ of individuals within the low and high probability of cognitive impairment groups. However, we were still uncertain of the probability of cognitive impairment for almost half of our sample in the intermediate group. We found that pairing the SDMT, a brief test of processing speed, with the MoCA improved classification for the indeterminate group, such that most cases in that group $(65 \%)$ were correctly identified. Examining individual subdomains of the MoCA did not significantly improve its ability to discriminate cognitive impairment for the indeterminate group.

Our findings confirm and extend those of Swartz and colleagues, ${ }^{14}$ who reported similar cut points (23 and 27) and a large indeterminate group in a sample of 400 individuals from a stroke clinic. The slightly different samples in the two studies (post-acute stroke vs. individuals referred to a stroke prevention clinic) suggest that the cut points are generalizable, at least within populations with vascular-related concerns. We expand on these previous findings by demonstrating improved diagnostic accuracy when adding a measure of processing speed to the MoCA.

Processing speed is affected in many neurological disorders ${ }^{8,27}$ and has been identified as a possible reason for the MoCA's reduced sensitivity for vascular-related cognitive impairment. $^{2,7}$ The addition of the SDMT to the MoCA directly addresses this issue by including a domain likely to be impaired in this population. This approach was first demonstrated by Dong and colleagues ${ }^{11}$ who demonstrated improved accuracy of VCI screening by supplementing the MoCA with the SDMT. Our study further refines this approach by introducing a staged method whereby the SDMT is administered to individuals scoring in the intermediate range on the MoCA (24-27) and whose cognitive status is therefore not certain after assessment with the MoCA alone. The SDMT also measures other cognitive domains in addition to processing speed, including divided attention, learning, working memory and complex visual scanning. ${ }^{10,27}$ Because the test uses both verbal and nonverbal materials, the SDMT incidental learning trial may be even more sensitive to subtle memory problems than other memory measures utilizing a single kind of material. Although some studies have reported impaired verbal and nonverbal learning in vascular dementia, ${ }^{28}$ the use of the incidental learning trial has not been implemented in other studies using the SDMT.7,11,28 
There are a number of clinical implications of our findings. Where clinical resources are limited and to avoid unnecessary referrals, employing an additional 90-second processing speed measure such as the SDMT can increase confidence in detecting cognitive impairment post-stroke. This approach is both practical and efficient as an addition to a screening test. When patients score in the intermediate range (24-27) on the MoCA and score more than 1.5 SDs below normative data on the SDMT coding, they are highly likely to have cognitive impairment and do not require further testing. The SDMT is an easily administered and quickly scored test with age-and education-corrected norms that are readily available and provide sensitive information that can be reliably used to triage patients. To optimize the use of the SDMT incidental learning component in this manner, there is a need for age- and education-corrected norms. Ideally when there are concerns about cognition, testing should occur after the acute phase, which is typically 3 months post-stroke, when cognition has stabilized and is less likely to fluctuate. ${ }^{15}$ The screen can be conducted in either specialized stroke clinics, general practitioner's office, or any setting where individuals who have had a stroke in the past ( $>3$ months) are now concerned about cognitive complaints and need to be triaged for more specialized care. In a clinical setting, false positives may pose a costly outcome when busy clinics and expensive treatments are used for individuals who do not need them. Similarly, false negatives can be detrimental in providing appropriate treatment for those who most need it. As such, the three-category approach to identify individuals with and without cognitive impairment directly addresses these issues by effectively triaging individuals who are at low, indeterminate, and high probability of cognitive impairment.

There are some caveats to our design that should be noted. First, participants may have been higher functioning than those typically seen in the general clinic by virtue of the study requirements: they had to have sufficient motor, language, hearing, and visual functioning to complete neuropsychological testing. Similarly, only individuals who scored $\geq 18$ on the MoCA were included, and as such we were unable to test diagnostic accuracy across the full range of cognition post-stroke. Additionally, we used a definition of cognitive impairment that includes those with even mild impairment, namely, $\geq 1.5 \mathrm{SD}$ on two or more tests in a single domain. Thus, there would be a higher prevalence of cognitive impairment in our sample than in other studies that use a more conservative definition (i.e., $\geq 2$ SD). Lastly, the cognitive domains used in our definition of cognitive impairment were based on a principal components analysis conducted on this sample, which restricts the generalizability of the results to other diseases.

\section{Conclusions}

The MoCA's sensitivity and specificity are maximized to higher than $90 \%$ by using two cut points to stratify post-stroke individuals into groups of low, indeterminate, and high probability of cognitive impairment. This is a practical and efficient method to increase certainty of classification for the indeterminate group while also expanding the domains assessed by pairing the MoCA with the SDMT. This process has the potential to improve the efficiency of limited and expensive resources, which is an important goal within the healthcare system.

\section{ACKNOWLEDGMenTs}

This work was completed on behalf of the Ontario Neurodegenerative Disease Research Initiative (ONDRI). The authors would like to acknowledge the ONDRI Founding Authors: Robert Bartha, Sandra E. Black, Michael Borrie, Dale Corbett, Elizabeth Finger, Morris Freedman, Barry Greenberg, David A. Grimes, Robert A. Hegele, Chris Hudson, Anthony E. Lang, Mario Masellis, William E. McIlroy, Manuel Montero-Odasso, David G. Munoz, Douglas P. Munoz, J.B. Orange, Michael J. Strong, Stephen C. Strother, Sean Symons, Maria Carmela Tartaglia, and Lorne Zinman.

\section{Sources of Funding}

This research was conducted with the support of the Ontario Brain Institute, an independent non-profit corporation, funded partially by the Ontario government. The opinions, results, and conclusions are those of the authors and no endorsement by the Ontario Brain Institute is intended or should be inferred. RHS receives personal support for research from a Clinician-Scientist Phase II Award from Heart \& Stroke and by the Department of Medicine at Sunnybrook HSC.

\section{Statement of Authorship}

KZ, AKT, JBR, RHS, MAB, and LT conceived and designed the study. KZ, AKT, JBR, KMS, and DB analyzed and interpreted the data. KZ drafted the manuscript and AKT, JBR, RHS, KMS, MAB, LT, PMM, DK, and DB revised the manuscript for intellectual content. BP, BL, DJS, DD, AH, JM, and The ONDRI Investigators contributed to the acquisition of data. All authors approved the manuscript.

\section{Disclosures}

None.

\section{REFERENCES}

1. Heart and Stroke Foundation. Disconnected: How Unseen Links Are Putting Us At Risk. 2019 Report On Heart, Stroke And Vascular Cognitive Impairment. Canada: Heart And Stroke. 2019. https:// www.heartandstroke.ca/-/media/pdf-files/canada/2019-report/ heartandstrokereport2019.ashx. Accessed November 10, 2019.

2. Chan E, Khan S, Oliver R, Gill S, Werring D, Cipolotti L. Underestimation of cognitive impairments by the Montreal Cognitive Assessment (MoCA) in an acute stroke unit population. J Neurol Sci. 2014;343(1-2):176-79. doi: 10.1016/j.jns.2014.05. 005 .

3. Nasreddine Z, Phillips N, BÃcdirian V, et al. The Montreal Cognitive Assessment, MoCA: A brief screening tool For mild cognitive impairment. J Am Geriatr Soc. 2005;53(4):695-99. doi: 10.1111/j.1532-5415.2005.53221.x.

4. Hachinski V, Iadecola C, Petersen R, et al. National institute of neurological disorders and stroke-Canadian stroke network vascular cognitive impairment harmonization standards. Stroke. 2006;37(9):2220-41. doi: 10.1161/01.str.0000237236.88823.47.

5. Price CC, Jefferson AL, Merino JG, Heilman KM, Libon DJ. Subcortical vascular dementia: Integrating neuropsychological and neuroradiologic data. Neurology. 2005;65(3):376-82. doi: 10.1212/01.wnl.0000168877.06011.15.

6. Cumming TB, Bernhardt J, Linden T. The Montreal Cognitive Assessment. Stroke. 2011;42(9):2642-44. doi: 10.1161/strokeaha. 111.619486.

7. Pendlebury S, Markwick A, Jager CD, Zamboni G, Wilcock G, Rothwell P. Differences in cognitive profile between TIA, stroke 
and elderly memory research subjects: A comparison of the MMSE and MoCA. Cerebrovasc Dis. 2012;34(1):48-54. doi: $10.1159 / 000338905$

8. Cees De Groot J, De Leeuw FE, Oudkerk, M, et al. Cerebral white matter lesions and cognitive function: the Rotterdam Scan Study. Ann Neurol. 2000;47(2):145-51. doi: 10.1002/15318249(200002) 47:2.

9. Pendlebury ST, Cuthbertson FC, Welch SJ, Mehta Z, Rothwell PM. Underestimation of cognitive impairment by Mini-Mental State Examination versus the Montreal Cognitive Assessment in patients with transient ischemic attack and stroke. Stroke. 2010;41(6):1290-93. doi: 10.1161/strokeaha.110.579888.

10. Smith A. Symbol Digit Modalities Test. Torrance, CA: Western Psychological Services; 1982.

11. Dong Y, Slavin MJ, Chan BP, et al. Improving screening for vascular cognitive impairment at three to six months after mild ischemic stroke and transient ischemic attack. Int Psychogeriatri. 2014;26(5):787-93. doi: 10.1017/S1041610213002457.

12. Nakas CT, Alonzo TA, Yiannoutsos CT. Accuracy and cut-off point selection in three-class classification problems using a generalization of the Youden index. Stat Med. 2010;29(28):2946-55. doi: 10.1002/sim.4044.

13. Attwood K, Tian L, Xiong C. Diagnostic thresholds with three ordinal groups. J Biopharm Stat. 2014;24(3):608-33. doi: 10. 1080/10543406.2014.888437.

14. Swartz RH, Cayley ML, Lanctôt KL, et al. Validating a pragmatic approach to cognitive screening in stroke prevention clinics using the Montreal Cognitive Assessment. Stroke. 2016;47(3):807-13. doi: 10.1161/strokeaha.115.011036.

15. Sivakumar L, Kate M, Jeerakathil T, Camicioli R, Buck B, Butcher K. Serial Montreal Cognitive Assessments demonstrate reversible cognitive impairment in patients with acute transient ischemic attack and minor stroke. Stroke. 2014;45(6):1709-15. doi: 10. 1161/STROKEAHA.114.004726.

16. Farhan SMK, Bartha R, Black SE, et al. The Ontario Neurodegenerative Disease Research Initiative (ONDRI). Canadian Journal of Neurological Sciences/Journal Canadien des Sciences Neurologiques. 2016;44(2):196-202. doi: 10.1017/cjn.2016.415.

17. McLaughlin PM, Sunderland KM, Beaton D, et al. The quality assurance and quality control protocol for neuropsychological data collection and curation in the Ontario Neurodegenerative Disease Research Initiative (ONDRI) study. Assessment. In press.
18. Sunderland KM, Beaton D, Fraser J, et al. The utility of multivariate outlier detection techniques for data quality evaluation in large studies: an application within the ONDRI project. BMC Med Res Methodol. 2019;19(1). doi: 10.1186/s12874-0190737-5.

19. Marian V, Blumenfeld HK, Kaushanskaya M. The Language Experience and Proficiency Questionnaire (LEAP-Q): Assessing language profiles in bilinguals and multilinguals. J Speech Lang Hear Res. 2007;50(4):940-67. doi: 10.1044/1092-4388(2007/067).

20. Rankin J. Cerebral vascular accidents in patients over the age of 60 : II. Prognosis. Scottish Medical Journal. 1957;2(5):200-15. doi: $10.1177 / 003693305700200504$.

21. Julayanont P, Nasreddine ZS. Montreal Cognitive Assessment (MoCA): Concept and clinical review. Cognitive Screening Instruments. 2017:139-95. doi: 10.1007/978-3-319-44775-9_7.

22. Uchiyama CL, Delia LF, Dellinger AM, et al. Longitudinal comparison of alternate versions of the symbol digit modalities test: Issues of form comparability and moderating demographic variables. Clin Neuropsychol. 1994;8(2):209-18. doi: 10.1080/ 13854049408401558.

23. Jak AJ, Bondi MW, Delano-Wood L, et al. Quantification of five neuropsychological approaches to defining mild cognitive impairment. Am J Geriatr Psychiatry. 2009;17(5):368-75. doi: 10. 1097/jgp.0b013e31819431d5.

24. James G, Witten D, Hastie T, Tibshirani R. An Introduction to Statistical Learning: with Applications in R. New York: Springer; 2017.

25. Brown MT, Wicker LR. Discriminant analysis. In: Tinsley HEA, Brown SD, ed. Handbook of applied multivariate statistics and mathematical modeling (pp.). San Diego, CA: Academic Press. 2000: 209-235. doi: 10.1016/B978-012691360-6/ 50009-4.

26. Dalrymple-Alford JC, Macaskill MR, Nakas CT, et al. The MoCA: Well-suited screen for cognitive impairment in Parkinson disease. Neurology. 2010;75(19):1717-25. doi: 10.1212/wnl. 0b013e3181fc29c9.

27. Lezak MD. Neuropsychological assessment. New York: Oxford University Press; 2012.

28. Sachdev PS, Brodaty H, Valenzuela MJ, et al. The neuropsychological profile of vascular cognitive impairment in stroke and TIA patients. Neurology. 2004;62(6):912-19. doi: 10.1212/01.wnl. 0000115108.65264.4b. 